\title{
EMPLEO DE FÓRMULAS INFANTILES ANTIRREGURGITACIÓN EN LACTANTES. EFECTO SOBRE LA DISPONIBILIDAD MINERAL
}

\author{
USE OF ANTI-REGURGITANT INFANT FORMULAS IN \\ UNWEANED BABIES. EFFECT ON MINERAL AVAILABILITY
}

\author{
Carlos A. González-Bermúdez., Carmen Frontela-Saseta, Patricia Peso-Echarri, \\ Rubén López-Nicolás, Carmen Martínez-Graciá
}

\begin{abstract}
Departamento de Tecnología de Alimentos, Nutrición y Bromatologia.
Facultad de Veterinaria. Universidad de Murcia. España.
\end{abstract}

\begin{abstract}
Gastroesophageal reflux (GER) is common in infants during the early months of life, as a result of a weak development of the regulatory mechanisms. Nevertheless, GER tends to disappear as infants grow. Nowadays, GER has become a common cause of pediatric consultation in the clinical practice, especially if it is followed by vomits. Sometimes, changing baby position while feeding seems to be a solution to reduce symptoms, while other times it is necessary manage the problem according to clinical practice guidelines. In these guidelines, the use of antiregurgitation infant formulas are included, which has been formulated with thickening agents and/or whey/casein ratio modification. Antiregurgitation milk products are available without pediatric prescription in chemists and other authorized establishments. Published studies on antiregurgitant infant formulas show some

contradictory conclusions about their use. A negative effect on mineral and micronutrient bioavailability has been proposed by some authors, in relation to the presence of thickening agents used as ingredients. Different aspects which require more research, related to the use of antiregurgitation infant formulas, have been included in this review with the aim of producing more effective and safety products for an especially vulnerable population, where an optimum feeding will lead to an adequate development and good health status in the future.
\end{abstract}

Key words: infant formula; gastroesophageal reflux; antiregurgitants; mineral availability.

Este trabajo fue recibido el 24 de Octubre de 2011 y aceptado para ser publicado el 18 de Noviembre de 2011.

\section{INTRODUCCIÓN}

El reflujo gastroesofágico se define como el movimiento retrógrado pasivo del alimento ingerido, desde el estómago al esófago (1-5). Los episodios de reflujo son frecuentes en los primeros meses de vida del lactante, con una incidencia del $40-60 \%$ en los 2 - 3 meses de edad, mostrando en la mayoría de las ocasiones, una tendencia natural a desaparecer hacia los $12-18$ meses de vida, despareciendo por completo en todos los niños a los 24 meses de edad (5-8). No obstante, si persiste en la edad preescolar o se manifiesta en niños mayores, se observan períodos de mejoría y recaída con una tendencia a persistir hasta la edad adulta en un $50 \%$ de los casos (Armas-Ramos et al., 2010). La mayoría de los episodios de reflujo gastroesofágico son asintomáticos, de breve duración y limitados a la porción distal del esófago, pudiendo o no acompañarse de vómito $(1,3)$. Este reflujo es considerado fisiológico, siempre y cuando formen parte del desarrollo y la función normales del tracto digestivo, sin consecuencias clínicas y repercusiones sobre la calidad de vida y el desarrollo del lactante (3, 5). Cuando estos episodios de reflujo van acompañados de vómito, suelen ser motivo de consulta pediátrica frecuente por la incomodidad y alarma que suscitan en los padres (9). Sin embargo, sólo en aquellos casos en los que se ve afectada la calidad de vida del lactante, por la frecuencia, duración, contenido y severidad de los episodios de reflujo, este proceso pasa a ser clasificado como patológico, denominándose enfermedad por reflujo gastroesofágico $(5,10,11)$. 


\section{Epidemiología y fisiopatología del reflujo gastroesofágico}

Diversos estudios han estimado una prevalencia del reflujo gastroesofágico del 40-65\% en lactantes de menos de 4 meses de edad $(1,6,11,12)$ no existiendo diferencias sustanciales con el sexo del lactante o con el tipo de alimentación (lactancia materna o fórmulas infantiles) (6).De todos ellos, solo un 20 - $24 \%$ son motivo de consulta pediátrica durante los primeros seis meses de vida $(1,13)$ debido a que los padres no perciben el vómito como un problema si no ocurre más de una vez al día, o si el lactante no manifiesta signos de dolor (12, 14). La regurgitación y vómito son, después del cólico y la constipación, la causa de consulta pediátrica por desórdenes gastrointestinales moderados más prevalente (6).

En el caso de los niños menores de un año, la enfermedad por reflujo gastroesofágico se suele encontrar asociada de forma secundaria a otras patologías, como malformaciones congénitas gastrointestinales, patologías neurológicas y cardiorespiratorias. Desde el punto de vista de su fisiopatología, el proceso por el cual el reflujo gastroesofágico tiene lugar, debemos entenderlo, como una compleja interacción de diferentes mecanismos implicados, algunos de ellos facilitadores del reflujo y otros protectores frente al mismo como las barreras anatómicas del esfínter esofágico,el diafragma y el ligamento freno esofágico (5).

La eficacia de este complicado sistema antirreflujo está limitada, por su inmadurez y limitado tamaño del esófago en el recién nacido y lactante pequeño (15). Con el desarrollo del niño y la evolución de estos órganos y sistemas involucrados, los episodios de reflujo gastroesofágico fisiológico desaparecen prácticamente en todos de los niños a los dos años de edad (5 - 8).

Se ha descrito que los niños que presentan una mayor frecuencia de episodios de reflujo, la porción esofágica intra-abdominal es prácticamente inexistente favoreciendose la propulsión del contenido gástrico hacia el esófago (14).

\section{Tratamiento del reflujo gastroesofágico}

Como se ha descrito, el reflujo gastroesofágico es un proceso complicado, donde intervienen diferentes factores, con una sintomatología más o menos acusada en función de la gravedad del proceso, siendo por ello esencial la evaluación por parte del pediatra, existiendo una guías publicadas en 2009 por los comités de las Sociedades Europea (ESPGHAN) y Norteamericana (NASSPGHAN) de Gastroenterología, Hepatología y Nutrición Pediátricas (16) para el diagnóstico y tratamiento del reflujo gastroesofágico del lactante. Estas guías recogen como recomendación en su tratamiento el empleo de sustancias espesantes añadidas a las fórmulas infantiles que se suministran a los lactantes, observándose una mejora en la sintomatología.

La leche humana es un alimento óptimo y completo que se va adaptando a las necesidades nutricionales del lactante durante los primeros 4 meses de vida. La leche materna, además de un adecuado aporte de nutrientes en formas altamente biodisponibles para el lactante, supone un aporte de células vivas (linfocitos y macrófagos), enzimas digestivas, inmunomoduladores y factores de crecimiento, importantes para la correcta alimentación y desarrollo del recién nacido $(17,18)$. No obstante, son muchos los factores que hacen que, en la actualidad, aproximadamente un $25 \%$ de madres recurren al empleo de fórmulas infantiles para la alimentación de los niños $(18,19)$.

Cuando la lactancia materna no es posible, se debe recurrir al empleo de fórmulas infantiles, formuladas para imitar la leche materna (20). Se han desarrollado fórmulas infantiles que suponen la alimentación fundamental del lactante y también destinadas a resolver algunos problemas digestivos menores, estando modificadas en su composición incluyéndose en la clasificación de fórmulas infantiles adaptadas (21). Dentro de este grupo de fórmulas adaptadas, encontramos las fórmulas antirregurgitación, especialmente formuladas con espesantes para aumentar su viscosidad y favorecer la retención del alimento en el estómago del lactante $(7,10,21)$.

El empleo de estas fórmulas antirregurgitación ha sido recomendado para el manejo de reflujo gastroesofágico frecuente, acompañado de vómito, que no presenta otras complicaciones en el niño (16). Este tipo de fórmulas no está recomendada en el caso de esofagitis, ya que no se han encontrado evidencias que demuestren que sean capaces de reducir los reflujos ácidos y, al tratarse de un alimento espeso, podrían alargar el tiempo de aclaramiento esofágico, agravando las lesiones (10, 11, 21, 22). A pesar de encontrar este tipo de fórmulas disponibles en el mercado, la ESPGHAN y el Comité de Nutrición de la Asociación Española de Pediatría (AEP) recomiendan el empleo de dietas con agentes espesantes únicamente en aquellos niños que presenten un retraso en el crecimiento derivado de una elevada frecuencia de episodios de reflujo gastroesofágico y vómitos, así como irritabilidad o trastornos del sueño, y siempre bajo supervisión médica $(10,15,23)$.

\section{Agentes espesantes y su uso en fórmulas infantiles antiregurgitación}

En los niños con regurgitación y vómitos frecuentes, tradicionalmente se ha recomendado el espesamiento de alimentos con el objetivo de aumentar la viscosidad y 
favorecer la retención en el estómago (21), siendo unas de las medidas empleadas la adición de harina de arroz a la fórmula infantil o a la leche, lo cual resultaba poco preciso y especialmente difícil para el caso de niños alimentados con leche materna (24).

En 1954, se publicó un trabajo que estudiaba un preparado comercial a base de harina de algarrobo, denominado Nestargel ${ }^{\circledR}$ para el tratamiento de lactantes con vómitos y regurgitaciones (25). Años más tarde, el mismo preparado comercial fue estudiado por Tausek \& Jurgensen (1957) (26). Desde entonces y hasta la actualidad, se han ido desarrollando fórmulas infantiles adaptadas con sustancias espesantes en su composición, indicadas para lactantes que presentan regurgitaciones y vómitos frecuentes sin otras complicaciones más graves $(10,7,21)$.

Los principales espesantes empleados en la elaboración de fórmulas antiregurgitación son la harina de semilla de algarrobo y los almidones pre-gelatinizados de maíz, patata o arroz $(6,21)$. El año 1992, la Comisión Europea, a través del Comité Científico para los Alimentos (27), aceptó la adición de almidones pre-gelatinizados a fórmulas infantiles con una concentración de máxima de $2 \%$ en fórmulas infantiles, sin que se pueda sobrepasar el $30 \%$ del contenido total de carbohidratos. Actualmente está vigente la Directiva 2006/141/CE (28), que especifica el empleo de almidones pre-gelatinizados dentro de los límites anteriormente citados. Con respecto a la harina de semillas de algarrobo (E-410), fue aceptada como un aditivo para la elaboración de alimentos infantiles con un fin médico especial el año 1997, no pudiendo emplearse en aquellos alimentos infantiles destinados a niños con buen estado de salud. Su empleo queda regulado por la Directiva 95/2/CE (29) del Parlamento Europeo y sus posteriores modificaciones, pudiendo adicionarse en una concentración máxima de $10 \mathrm{~g} / \mathrm{L}$ desde el nacimiento, en productos destinados a reducir el reflujo gastroesofágico.

La adición de espesantes a las fórmulas antiregurgitación, tiene por objetivo aumentar la viscosidad para el tratamiento del reflujo gastroesofágico no complicado (18). La viscosidad se define como una medida reológica cuantitativa de la resistencia de un fluido debida al rozamiento de las partículas del mismo con la superficie por la que fluye (30) es una característica clave para el diseño y actividad de este tipo de fórmulas. No obstante, los estudios sobre el efecto de la adición de estos agentes espesantes sobre la viscosidad de las fórmulas infantiles son escasos (18). Se han realizado medidas de la viscosidad in vitro, simulando para ello un proceso digestivo mediante la adición de enzimas gástricas y ácido clorhídrico. Vanderhoof et al., (2003) (30) evaluaron el efecto de la variación del $\mathrm{pH}$ sobre la viscosidad de una fórmula infantil comercial estándar a base de leche de vaca, frente a su homóloga antiregurgitante, en la cual se había sustituido el $30 \%$ de lactosa con almidón de arroz pregelatinizado (2,3 g de almidón/100mL). Durante el estudio, se evaluó la viscosidad de la fórmula estándar, a la cual se le adicionó una cucharada de cereal de arroz previo a su reconstitución observándose que conforme descendió el valor de $\mathrm{pH}$, la viscosidad de las fórmulas a las que se les añadió el espesante incrementó, siendo este incremento más marcado cuando el $\mathrm{pH}$ descendió de un valor de $6-5,5$.

En el caso de la fórmula infantil a la que se le añadió el espesante antes de su reconstitución, la viscosidad de la fórmula antes de la variación de $\mathrm{pH}$ aumentó cerca de 200 veces, en comparación con fórmula sin espesante. En el caso de la fórmula comercial con antiregurgitante, el incremento inicial de la viscosidad fue mucho menor, aproximadamente de unas 60 veces de la viscosidad inicial de la fórmula sin espesante. Cuando se estudió la evolución de la viscosidad con respecto al descenso del $\mathrm{pH}$, se observó como el aumento brusco de la viscosidad que se mantuvo estable para el caso de la fórmula a la que se le adicionaron los cereales, mientras que para la fórmula con antiregurgitante comenzó a disminuir al llegar a un valor de $\mathrm{pH}$ de 4,5. Vanderhoof et al., (2003) (30) concluyeron así que, la adición de cereales a una fórmula convencional supone un incremento inicial de la viscosidad que puede dificultar el paso del homogeneizado a través de la tetina del biberón, mientras que la fórmula antiregurgitante se mantuvo más fluida durante la alimentación del lactante. Otro aspecto que señalan de importancia es el descenso de viscosidad que se produce en la fórmula con antiregurgitante al alcanzar un valor de $\mathrm{pH}$ 4,5 frente a la fórmula con cereal, lo cual es importante en el caso de aparición de reflujos ácidos y de elevada viscosidad que pueden ver aumentado el tiempo de permanencia del material en contacto con la mucosa esofágica, agravando problemas de esofagitis. En el caso de la fórmula antiregurgitante, al sustituir la lactosa por el almidón precocido, la composición nutricional de la misma es más semejante a la de la leche materna (30).

Respecto a la adición de cereales a fórmulas infantiles, la ESPGHAN recomienda no introducir los cereales en el calendario de alimentación del lactante antes de los 4 meses de edad. Hay estudios que afirman que la adición de cereales para espesar la fórmula en el tratamiento del reflujo gastroesofágico, aumenta la ingesta calórica aproximadamente $25 \%$, lo que puede producir un aumento ponderal del niño (2).

En el estudio, de Infante-Pina et al., (2010) (18) se determinó el comportamiento reológico de diferentes 
fórmulas comerciales con antiregurgitante presentes en el mercado español, con diferente espesante añadido y a distinta concentración (almidón de patata y maíz, almidón de arroz, harina de semilla de algarrobo y almidón de maíz), comparando los resultados con una fórmula comercial control y con la fórmula control a la que se le adicionó un $10 \%$ de cereales sin gluten. Los autores concluyeron que sólo algunas fórmulas infantiles elaboradas con antiregurgitantes eran capaces de aumentar la viscosidad por encima de los valores que se alcanzan con la adición de una cucharada de cereales sin gluten. Concluyen que existen tres factores que influyen de forma decisiva en el comportamiento reológico de la fórmula infantil:

- Gelatinización de los almidones: en condiciones gástricas fisiológicas, el $\mathrm{pH}$ y el aumento de temperatura producen una gelatinización del almidón que se hincha de manera irreversible reorganizando sus gránulos. Este hecho impediría un aumento significativo de la viscosidad y su mantenimiento a lo largo de todo el proceso digestivo. En el caso de la fórmula a la que se adiciona $10 \%$ de cereales, la alta viscosidad obtenida se justifica por la elevada concentración de almidones, que podría compensar la gelatinización de los mismos.

- Proporción de caseína: La caseína de la leche se agrupa formando parte de micelas con elevado peso molecular. $\mathrm{Al}$ descender el $\mathrm{pH}$ a valores cercanos a 4 unidades, estas micelas floculan, formando un coágulo que dificulta la digestión. Una mayor concentración de caseína podría, evitar el descenso de la viscosidad, al dificultar la acción enzimática. En el caso de una fórmula infantil con un contenido en caseína estándar y a la que se le adiciona cereal de arroz para espesarla durante su reconstitución, ésta resulta ser menos eficaz en el manejo del reflujo gastroesofágico que una fórmula en la cual se sustituye parte de la lactosa por almidones pre-gelatinizados y se aumentó el contenido en caseina, justificando este efecto antiregurgitante a través de la floculación de la caseína (7).

- Concentración del espesante: En aquellas fórmulas en las que el cociente proteico es similar y el tipo de espesante es el mismo la viscosidad conseguida estuvo en función de la concentración de espesante. Lo más eficaz para conseguir una viscosidad adecuada en una fórmula antiregurgitante, descartando el empleo de la adición de cereales a una fórmula comercial, sería la adición de harina de algarrobo y la presencia de niveles elevados de caseína. Es necesario establecer el límite de viscosidad máxima para este tipo de fórmulas, indicando que una viscosidad superior a 100 centipoises podría comprometer el aclaramiento esofágico y favorecer la aparición de esofagitis en el lactante (18).

\section{Eficacia de las fórmulas infantiles $\mathrm{AR}$ en la alimentación de lactantes con RGE no complicado}

El objetivo de las fórmulas infantiles es sustituir o complementar a la lactancia materna, cuando esta no es posible, consiguiendo un adecuado desarrollo del niño, prevenir deficiencias nutricionales y un buen desarrollo de sus funciones inmunológicas (8). En el caso de las fórmulas antiregugitantes, además deben ser capaces de reducir los síntomas asociados al reflujo gastroesofágico no complicado (31). Pese a que el empleo de este tipo de fórmulas ha sido recomendado para el manejo del reflujo gastroesofágico no complicado (16), existe una gran controversia sobre su efectividad $(4,13,32)$.

Diversos estudios han evaluado la eficacia de este tipo de fórmulas infantiles y de los distintos agentes espesantes en la reducción de los síntomas asociados al reflujo gastroesofágico en lactantes en el vaciado gástrico y en los episodios de reflujo (2, 30, 32, 33). Horvath et al., (2008) (4) recopilaron los resultados de trabajos publicados a lo largo de distintos años para evaluar la efectividad de espesantes añadidos a fórmulas infantiles, concluyendo que el empleo de agentes espesantes es capaz de reducir la frecuencia de aparición de episodios de regurgitación y vómito en la mayoría de los casos. Sin embargo, aquellos estudios donde se evaluó la eficacia de los agentes espesantes mediante medida de su impedancia-intraluminal mostraron que, pese a reducir la sintomatología asociada, no produjeron una reducción significativa en el número de episodios de reflujo gastroesofágico $(13,33,34)$. Este hecho puede ser explicado porque entre los agentes espesantes empleados se empleó la harina de semilla de algarrobo, presentando ésta a mayor concentración, mayor densidad calórica y mayor osmolaridad, retrasando el tiempo de vaciamiento gástrico, pudiéndose también afectar la variabilidad fisiológica de cada individuo del estudio $(33,34)$. Según éstos últimos autores, durante la primera mitad del período postpandrial prevalecen principalmente los episodios de reflujo gastroesofágico no ácido (89-84\%), mientras que durante la segunda mitad del período postpandrial, la prevalencia de reflujo gastroesofágico ácido aumenta en un $65-75 \%$. Esto debe tenerse en cuenta a la hora de determinar la concentración del espesante a emplear en las fómulas infantiles ya que, un retraso en el tiempo de vaciamiento gástrico se traduce en un incremento de los episodios de reflujo (32) pudiendo suponer un agravamiento del proceso.

En relación a la ganancia de peso de los niños alimentados con las fórmulas infantiles antirregurgita- 
ción, se ha descrito un ligero incremento del mismo con respecto a los niños alimentados con fórmulas infantiles estándar, atribuyendo a una mayor ingesta calórica y a un incremento en las horas de sueño y tranquilidad del lactante $(2,4)$.

\section{Efectos negativos de las fórmulas antiregurgitación sobre la biodisponibilidad mineral}

La composición de la leche materna la hace óptima para una adecuada biodisponibilidad de minerales fundamentales en la nutrición del niño encontrándose en su mayor parte unidas a proteínas que actúan como transportadoras $(35,36)$. La biodisponibilidad puede ser definida como la proporción de un nutriente que puede ser utilizada por el organismo a través del metabolismo. En el caso de los micronutrientes, deben ser liberados del alimento y solubilizados en el intestino, permitiendo su absorción por las células intestinales. Este proceso es complejo, y en él intervienen multitud de factores fisiológicos, junto a otros dependientes de la propia matriz alimentaria (35). Las fórmulas con antiregurgitantes incluyen en su composición carbohidratos no digeribles, asociados a su ingesta, se ha descrito una potencial disminución en la absorción intestinal de calcio, hierro y cinc en el intestino por sus propiedades quelantes $(4,21,35)$.

Los requerimientos de minerales que tiene el lactante no se mantienen estables durante su desarrollo, sino que van modificando en las distintas etapas, siendo los Requerimientos Diarios Recomendados establecidos para el $\mathrm{Ca}, \mathrm{Fe}$ y $\mathrm{Zn}$ durante los tres primeros años de vida, los que se muestran en la tabla 1 (38).

Los estudios sobre cómo afectan los espesantes empleados en las fórmulas antirregurgitación la biodisponibilidad mineral son escasos en la actualidad. Se ha evaluado este efecto in-vitro y en modelos animales, ante la dificultad para realizar este tipo de estudios in-vivo en humanos, por cuestiones éticas y técnicas (31).

Entre los estudios revisados de Bosscher et al.,
$(2000,2001))(39,40)$ son los que más luz han arrojado a estas cuestiones. Se empleó un método in-vitro optimizado y validado de digestión y diálisis para simular el proceso de digestión gastrointestinal y determinar la proporción de mineral dializado tras dicho proceso. Ésta proporción se ha calculado con respecto a la cantidad de mineral total presente en las fórmulas estudiadas. Entre los agentes espesantes, se analizaron la harina de semilla de algarrobo y los almidones pre-gelatinizados de arroz, evaluando también el efecto de la alteración del cociente suero/caseína en dichas fórmulas. Los resultados se compararon con los de fórmulas estándar y la leche materna.

En lo referente a la harina de algarrobo, estos estudios encontraron que su adición a la fórmula infantil ejerció un efecto quelante sobre el calcio y, especialmente, sobre el hierro y el cinc, reduciendo la biodisponibilidad mineral bajo condiciones gastrointestinales simuladas. Este efecto se vió acentuado cuando las fórmulas fueron modificadas en su relación suero:caseína. Con respecto a los almidones pregelatinizados, no encontraron diferencias significativas en la biodisponibilidad de hierro, calcio y cinc con respecto a las fórmulas de referencia y la leche materna.

La digestibilidad de los almidones modificados ha resultado ser excelentes ( $>95 \%)$ en niños de $1-3$ meses de edad, por lo que no se debería ver afectada de modo negativo la biodisponibilidad mineral. Sin embargo, la digestibilidad de la harina de algarrobo es muy baja, llegando apenas digerida al colon. Este hecho hace que la absorción intestinal se pueda ver disminuida (Bosscher et al., 2000). En el caso de algunas fórmulas infantiles en las que la relación suero:caseína está alterada con predominio de la caseína se puede originar problemas en la digestión del niño que agrava el efecto negativo sobre la absorción intestinal de minerales $(6,30,35,41)$.

Al comparar estos resultados con escasos estudios que se han realizado en adultos y niños sobre el efecto de estos agentes espesantes en la absorción mineral

\section{TABLA 1}

\section{Requerimientos diarios recomendados (RDR) de Ca, Fe y Zn (mg/día) durante los tres primeros años de vida.}

$\begin{array}{lccc}\text { MINERALES } & \mathbf{0}-\mathbf{6} \text { meses } & \mathbf{6 - 1 2} \text { meses } & \mathbf{1} \mathbf{- 3} \text { años } \\ \text { Calcio } & 400 & 600 & 800 \\ \text { Hierro } & 6 & 10 & 10 \\ \text { Zinc } & 5 & 5 & 10\end{array}$


(42) comprobaron que tras un estudio antropométrico y nutricional en niños alimentados con fórmulas-infantiles con harina de algarrobo que no había diferencias significativas en los niveles de calcio, hierro y cinc con niños alimentados con fórmulas control. Estos resultados estuvieron de acuerdo con los obtenidos por Levtchenko et al.,(42) y Behall et al., (1987) (43) que no encontraron ningún efecto negativo sobre el balance mineral tras adicionar harina de algarrobo en una dieta basal de adultos.

Bosscher et al., (2001) (40) justificaron diferencias entre ambos modelos, al indicar que en el modelo in-vitro no se pueden reproducir mecanismos compensatorios que ocurrirían en el organismo vivo como la reducción de la excreción urinaria de minerales o el efecto fermentativo de la flora del colon sobre las fibras no digeribles, liberando parte de los minerales quelados que serían absorbidos a estos niveles.

La posibilidad de fortificar las fórmulas infantiles con formas minerales altamente biodisponibles puede ser considerada una buena estrategia. Perales et al., (2006) (44), realizaron un estudio in-vitro sobre células de colon humano para evaluar el efecto de la fortificación de leche con calcio y su efecto sobre la biodisponibilidad de otros minerales esenciales, encontrando que un incremento en los niveles de calcio se traduce en un aumento en la biodisponibilidad del mismo sin modificar la absorción de cinc.

La investigación sobre fórmulas infantiles con el objetivo de conseguir una mayor semejanza con la leche materna continúa actualmente progresando, y está encaminada a conseguir alimentos completos y funcionalmente adecuados. Las fórmulas antirregurgitación pueden ser una interesante herramienta para el manejo del reflujo gastroesofágico no complicado del lactante, sobre todo cuando la frecuencia de estos episodios, así como los síntomas secundarios asociados puedan repercutir en la salud y calidad de vida del neonato. No obstante, las fórmulas infantiles antirregurgitación aunque suponen una alternativa al espesamiento tradicional con harina de cereal se hace necesaria la investigación de los diferentes aspectos asociados a este tipo de fórmulas, tanto en lo referente a los diferentes tipos de espesantes utilizados, como sobre posibles consecuencias que puedan tener sobre la salud del lactante.

Con respecto a su formulación y características reológicas, parece ser que el empleo de la harina de algarrobo permite un mayor espesamiento que los almidones pregelatinizados, siendo al mismo tiempo más resistente a los procesos digestivos, sobre todo si este ingrediente se asocia con una modificación de la proporción suero:caseína ya que el precipitado de caseínas dificulta la acción de las enzimas digestivas. Sería conveniente, por lo tanto, fijar el valor de viscosidad que se considere efectivo en el tratamiento de los episodios de reflujo no complicado del lactante, así como cuantificar las modificaciones asociadas a este parámetro en relación a las concentraciones de espesante empleadas fijando, de este modo, la mínima concentración de cada ingrediente que sería efectiva en el manejo del proceso.

Respecto de su efectividad en el tratamiento del reflujo gastroesofágico, consideramos interesante el empleo de ingredientes que consigan un espesamiento efectivo y estable durante todo el proceso digestivo. No obstante, un espesamiento del contenido gástrico conlleva un retraso en el vaciamiento del estómago y en el aclaramiento esofágico. Teniendo en cuenta que, es precisamente en la segunda mitad del proceso digestivo cuando aumenta la frecuencia de episodios de reflujo, supondría un mayor riesgo de una exposición prolongada de la mucosa esofágica a ácidos y enzimas gástricos. Se debería plantear, por lo tanto, si el empleo de harina de algarrobo o de almidones pregelatinizados es más aconsejable, sabiendo que estos últimos tienen una mejor digestibilidad y permiten un descenso más favorable de la viscosidad del contenido gástrico al final del proceso digestivo.

Se hace necesario investigar si la harina de algarrobo, al ser más resistente al proceso digestivo que los almidones pregelatinizados, podría tener un efecto añadido sobre la microflora intestinal del lactante y si este efecto sería beneficioso o perjudicial para la salud del lactante, ya que una fermentación excesiva podría repercutir en la aparición de otros procesos como cólicos.

En último lugar, la harina de algarrobo parece tener un efecto negativo sobre la disponibilidad in-vitro del calcio y hierro, y menos evidente sobre la del cinc. Dicho efecto se incrementa al modificar la relación suero:caseína, por la acción estabilizadora de los precipitados de caseína sobre acción enzimática digestiva . Este hallazgo no ha sido observado al evaluar los almidones pre-gelatinizados. Sin embargo, al evaluar ambos ingredientes in-vivo, en los estudios realizados hasta lo momento, no se han encontrado estos efectos negativos, debiendo considerar el papel que las fermentaciones bacterianas tendrían sobre la digestión de los espesantes y la liberación de los minerales retenidos en las etapas previas de la digestión gastrointestinal.

\section{CONCLUSIONES}

Ante la importancia de una adecuada alimentación del lactante, es necesario una investigación más profunda sobre el empleo y la formulación de fórmulas infantiles antirregurgitación. Se debe contemplar, un perfeccionamiento en la metodología in-vitro emplea- 
da hasta el momento, añadiendo fases de simulación de fermentación intestinal microbiana y la necesidad de su complementación con estudios in-vivo para que los resultados obtenidos sean un mejor reflejo de la población a la que va destinada. Mientras que los resultados no sean más concluyentes, el empleo de este tipo de fórmulas debería estar supervisado siempre por especialistas evitando su libre acceso en farmacias o tiendas especializadas.

\section{RESUMEN}

Las regurgitaciones gastroesofágicas en el lactante constituyen un proceso frecuente en los primeros meses de vida, fruto de la inmadurez de algunos mecanismos fisiológicos que regulan este proceso, con tendencia a desaparecer conforme avanza el desarrollo. Constituyen un motivo de consulta pediátrica frecuente, sobre todo cuando se acompañan de vómitos. En ocasiones, las recomendaciones posturales a adoptar durante la alimentación son suficientes para contribuir a mejorar la sintomatología. Cuando esto es insuficiente, existen protocolos de intervención en nutrición infantil como el diseño de fórmulas antirregurgitación, que contienen sustancias espesantes y/o modificaciones en la relación suero:caseína. Las fórmulas antirregurgitación se encuentran disponibles en farmacias y otros establecimientos de venta autorizados, sin que sea necesaria una prescripción médica para su adquisición. Estudios realizados sobre el empleo de fórmulas infantiles para el manejo pediátrico del reflujo gastroesofágico muestran ciertas contradicciones respecto a una recomendación sobre su uso. Algunos autores destacan que puede aparecer un efecto negativo sobre la biodisponibilidad mineral y de otros micronutrientes como consecuencia de los agentes espesantes utilizados como ingredientes. En la presente revisión se abordan los aspectos que, sobre el empleo de fórmulas infantiles antiregurgitación, precisan de mayor investigación para permitir elaborar fórmulas antirregurgitación eficaces y totalmente seguras para una población que es especialmente vulnerable, y en la que una correcta alimentación permite un adecuado desarrollo y buen estado de salud en la etapa adulta.

Palabras clave: fórmula infantil; reflujo gastroesofágico; antirregurgitantes; disponibilidad mineral

Dirigir la correspondencia a:

Señor

Carlos A. González-Bermúdez

Departamento de Tecnología de Alimentos,

Nutrición y Bromatología.

Facultad de Veterinaria
Universidad de Murcia

Campus de Espinardo

30100. Nurcia, España

Correo electrónico: cagb1@um.es

Teléfono: 868887985

Agradecimientos: A la Fundación Séneca por el proyecto de investigación: "Fórmulas infantiles antirregurgitación. Estudio de la bioaccesibilidad mineral y del efecto prebiótico" (11978/PI/09) y por la beca 15001/ BPS/10 concedida a C.A. González-Bermúdez.

\section{BIBLIOGRAFÍA}

1. Hyman PE, Milla PJ, Benninga MA, Davidson GP, Fleisher DF, Taminiau J. Childhood Functional Gastrointestinal Disorders: Neonate/Toddler. Gastroenterology 2006; 130: 1519 - 26.

2. Chao HC, Vandenplas Y. Effect of cereals-thickened formula and upright positioning on regurgitation, gastric emptying, and weight gain in infants with regurgitation. Nutrition 2007; 23: $23-8$.

3. Hegar B, Rantos R, Firmansyah A, De Schepper J, Vandenplas Y. Natural evolution of infantile regurgitation versus the efficacy of thickened formula. $J$ Pediat. Gastr Nutr. 2008; 47: 26 - 30.

4. Horvath A, Dziechciarz P, Szajewska H. The Effect of Thickened-Feed Interventions on Gastroesophageal Reflux in Infants: Systematic Review and Meta-analysis of Randomized, Controlled Trials. Pediatrics 2008; 122(6): 1268 - 77.

5. Martín de Capri J. Reflujo Gastroesofágico. Boletín de la sociedad de Pediatría de Aragón, La Rioja y Soria 2009; 39: 20 -5.

6. Vandenplas Y, Lifshitz JZ, Orenstein S, Lifshitz CH, Shepherd RW, Casaubón PR, Muinos WI, et al. Nutritional Management of Regurgitation in Infants. J Am Coll Nutr 1998; 17(4): 308 - 16.

7. Ramirez-Mayans J, Palacio-Del Carmen L, Cervantes-Bustamante $\mathrm{R}$, Mata-Rivera N, Pina-Romero $\mathrm{N}$, Zarate-Mondragon F, Gelis-Vieitez P, et al. Nutritional management of Children with Gastroesophageal Reflux: A Comparaison of Two Different Formulas. Internat Pediatr 2003; 18(2): 78 - 82.

8. García-Onieva M. Lactancia artificial: técnica, indicaciones, fórmulas especiales. Pediatría Integral 2007; XI(4): 318 - 26.

9. Vandenplas Y, Belli D, Benhamou S, Cadranel JP, Cezard S, Cucchiara C, Dupont C, et al. A critical appraisal of current management practices for infant regurgitation - recommendations of a working party. Eur J Pediatr. 1997; 156: 343 - 57.

10. Aggett PJ, Agostoni C, Goulet O, Hernell O, Ko- 
letzko B, Lafeber HL, Michaelsen K.F. Milla P., Rigo J; Weaver Lt. Antireflux or Antirregurgitation Milk Products for infants and Young Children: A commentary by the ESPGHAN Committee on Nutrition. J Pediatr Gastr Nutr. 2002; 34: 496 - 8.

11. Kostovski A. Gastro-oesophageal Reflux Disease. Paediatr Croat, 2006; 50(1): 93 - 102.

12. Nelson SP, Chen EH, Syniar GM, Christoffel KK. Prevalence of symptoms of gastroesophageal reflux during infancy. Arch Pediatr Adoles, 1997; 15: $569-72$.

13. Vandenplas Y. Thickened Infant Formulas Does What It Has To do: Decrease Regurgitation. Pediatrics 2009; 123(3): $549-50$.

14. Rudolph C, Mazur L, Liptak G, Baker R. Pediatric Gastroesophageal Reflux Clinical Practice Guidelines. J Pediatr Gastr Nutr 2001; 32(2):1 - 31.

15. Armas-Ramos H. Reflujo Gastroesofágico y en niños. En. Conoreso Nacional AEP 2008 Disponible en. $\mathrm{http \cdot //wwy.congresoaep.org/2008/agenda/}$

16. ESPGHAN/NASPGHAN. Pediatric Gastroesophageal Reflux Clinical Practice Guidelines: Joint Recommendations of the North American Society for Pediatric Gastroenterology, Hepatology, and Nutrition (NASPGHAN) and the European Society for Pediatric Gastroenterology, Hepatology, and Nutrition (ESPGHAN). J Pediatr Gastr Nutr. 2009; 49: 498 - 547.

17. Hernandez-Aguilar MT, Aguayo-Maldonado J. La lactancia materna. Cómo promover y apoyar la lactancia materna en la práctica pediátrica. Recomendaciones del Comité de Lactancia de la AEP. An Pediatr (Barc) 2005; 63(4): 340 - 56.

18. Infante-Pina D., Lara-Villosalada F., López-Ginés G., Morales-Hernández ME. Estudio del comportamiento reológico in vitro de las fórmulas antirregurgitación. An Pediatr 2010; 72(5):302-8.

19. Gage H, Morgan J, Williams P, Schmid M, Laitenen K, Von Rosen J, Koletzko B, et al. Infant feeding intentions of new mothers in five European Countries. Pr Nutr Soc. 2010; 69.

20. Joeckel RJ., Phillips SK. Overview of Infant and Pediatric Formulas. Nutr Clin Practice 2009; 24(3): $356-62$.

21. Ferrer-Lorente B., Vitoria-Miñana I., Dalmau-Serra J. Indicaciones para fórmulas lácteas especiales: fórmulas para problemas "menores", fórmulas sin lactosa y fórmulas de proteína de soja. Acta Pediatr Esp 2009; 67(7): 333 - 7.

22. Galicia-Poblet G. Manejo del reflujo gastroesofágico en la infancia. Boletín Farmaco-terapéutico de
Castilla la Mancha 2009; 10(3).

23. Ballabriga A., Moya M., Bueno M., Cornella J., Dalmau J., Doménech R., et al. Indicaciones de las fórmulas antirregurgitación. Comité de $\mathrm{Nu}-$ trición de la AEP. An Españoles Pediatr 2000; 52: $369-71$.

24. Rode H, Millar AJV, Brown RA, Melis J. Current concepts in the management of gastroesophageal reflux in infants. SAMJ 1998; 88(10): $1328-33$.

25. Gross H. The treatment of vomiting in infants with carob flour (Nestragel). Wien Med Wochenschr 1954; 104: $885-6$.

26. Tausek F, Jurgensen O. Clinical and roentgenological results of Nestragel treatment of habitual vomiting in infant. Munch Med wochenschr 1957; 99: $927-8$.

27. Commission of The European Communities, Scientific Committee For Food. Opinion on certain additives for use in infant formula, follow-up on formula and weaning foods.106th Meeting of the SCF on the 21 March 97, 1992 Disponible en. http.//ec.europa. eu/food/fs/sc/oldcomm7/out06_en.html

28. Directiva 2006/141/Ce De La Comisión De 22 De Diciembre De 2006 relativa a los preparados para lactantes y preparados de continuación y por la que se modifica la Directiva 1999/21/CE. DO L 401 de 30.12.2006: 1-39.

29. Directiva 95/2/Ce Del Parlamento Europeo Y Del Consejo De 20 De Febrero De 1995, relativa a aditivos alimentarios distintos de los colorantes y edulcorantes. DO L 61 de 18.3.1995: 1 - 68.

30. Vanderhoof JA, Moran JR, Harris CL, Merkel KL, Orenstein SR. Efficacy of a Pre-thickened Infant Formula: A Multicenter, Double-Blid, Randomized, Placebo-Controlled Parallel Group Trial in 104 Infants with Symptomatic Gastroesophageal Reflux. Clin Pediatr 2003; 42: 483 - 95.

31. Agostoni C. Antireflux or antiregurgitation milk products for infants and young children: a commentary by the ESPGHAN Committee on Nutrition. Acta Pædiatr 2004; 93: 456.

32. Miyazawa R, Tomomasa T, Kaneko H, Morikawa A. Effect of formula thickened with locust bean gum on gastric emptying in infants. J Pædiatr Child Health 2006; 42: 808 - 12 .

33. Corvaglia L., Ferlini M., Rotatori R., Paoletti V., Alessandroni R., Gocchi G., Faldella G. 2006. Starch thickening of human milk is ineffective in reducing the gastroesophageal reflux in preterm infants: A crossover study using intraluminal impedance. J Pediatr, 2006; $265-8$.

34. Wenzl T., Schneider S., Scheele F., Silny J., Hei- 
mann G., Skopnik H. Effects of thickened feeding on gastroesophageal reflux in infants: a placebo controlled crossover study using intraluminal impedance. Pediatrics 2003; 111: $161-5$.

35. Bosscher D., Van Caillie-Bertrand M., Van Cauwenbergh R., Deelstra H. Availabilities of Calcium, Iron and Zinc from Infant Formulas is Affected by Soluble Dietary Fibers and Modified Starch Fractions. Nutrition 2003; 19: $641-645$.

36. Almeida AA, Lopes MPVC, Silva MSA, Barrado E. Trace elements in human milk: Correlation with blood levels, inter-element correlations and changes in concentration during the first month of lactaction. J Trac Elem Med Biol 2008; 22: 196 - 205.

37. Savino F, Muratore M, Silvestro L, Oggero R, Mostert M. Allergy to carob gum in an infant. J Pediatr Gastr Nutr, 1999; 29(4): 475 - 6.

38. NRC, National Research Council. Nutrient Requirements of Recommended dietary allowances, 1991; Washington DC, National Academic Press.

39. Bosscher D, Van Caillie-Bertrand M, Van Dyck K, Robberecht H, Van Cauwenbergh R, Deelstra $H$. Thickening infant formula with digestible and indigestible carbohydrate: Availability of calcium, iron and zinc in vitro. J Pediatr Gastr Nutr. 2000; 30(4): $373-8$.

40. Bosscher D, Van Caillie-Bertrand M, Deelstra H. Effect on thickening agents, based on soluble dietary fiber, on the availability of calcium, iron and zinc from infants formulas. Nutrition 2001; 17: $614-8$.

41. Xinias I., Spiroglou K., Demertzidou V., Karatza E., Panteliadis C. An antiregurgitation Milk Formula in the Management of Infants with Mild to Moderate Gastroesophgeal Reflux. Curr Ther Res 2003; 64(4): $270-8$.

42. Levtchenko A, Hauser B, Vandenplas Y. Anthropometry and nutritional value of a whey adapted formula or a thickened AR formula in term born infants. J Pediatr Gastr Nutr 1997; 24: 491.

43. Behall K., Scholfield D., Lee K., Powell A., Moser P. Mineral balance in adult men: Effect of four refined fibers. Am J Clin Nutr, 1987; 46: 307 - 14.

44. Perales S., Barberá R., Lagarda M.J., Farré R. Fortification of milk with calcium: Effect on Calcium Bioavailability and interactions with Iron and Zinc. J Agric Food Chem, 2006; 54: 4901 - 06. 